\title{
Blurring the boundaries between frame-based and frameless stereotaxy: feasibility study for brain biopsies performed with the use of a head-mounted robot
}

\author{
*Florian Grimm, MD,' Georgios Naros, MD, ${ }^{1}$ Angelika Gutenberg, MD, ${ }^{2}$ Naureen Keric, MD, ${ }^{2}$ \\ Alf Giese, MD, ${ }^{2}$ and Alireza Gharabaghi, MD'1 \\ 1Division of Functional and Restorative Neurosurgery, Department of Neurosurgery, Eberhard Karls University, Tuebingen; and \\ 2Department of Neurosurgery, Johannes Gutenberg University, Mainz, Germany
}

\begin{abstract}
OBJECT Frame-based stereotactic interventions are considered the gold standard for brain biopsies, but they have limitations with regard to flexibility and patient comfort because of the bulky head ring attached to the patient. Frameless image guidance systems that use scalp fiducial markers offer more flexibility and patient comfort but provide less stability and accuracy during drilling and biopsy needle positioning. Head-mounted robot-guided biopsies could provide the advantages of these 2 techniques without the downsides. The goal of this study was to evaluate the feasibility and safety of a robotic guidance device, affixed to the patient's skull through a small mounting platform, for use in brain biopsy procedures.

METHODS This was a retrospective study of 37 consecutive patients who presented with supratentorial lesions and underwent brain biopsy procedures in which a surgical guidance robot was used to determine clinical outcomes and technical procedural operability.

RESULTS The portable head-mounted device was well tolerated by the patients and enabled stable drilling and needle positioning during surgery. Flexible adjustments of predefined paths and selection of new trajectories were successfully performed intraoperatively without the need for manual settings and fixations. The patients experienced no permanent deficits or infections after surgery.

CONCLUSIONS The head-mounted robot-guided approach presented here combines the stability of a bone-mounted set-up with the flexibility and tolerability of frameless systems. By reducing human interference (i.e., manual parameter settings, calibrations, and adjustments), this technology might be particularly useful in neurosurgical interventions that necessitate multiple trajectories.
\end{abstract}

http://thejns.org/doi/abs/10.3171/2014.12.JNS141781

KEY WORDS stereotaxy; frameless brain biopsy; frame-based brain biopsy; robotics; neuronavigation; tumor; oncology; diagnostic and operative techniques

A LTHOUGH brain biopsies generally involve minimal tissue disruption, eloquent regions and vascular structures must be avoided to minimize the impact on patient quality of life. To execute accurate planning of such trajectory-based procedures, most neurosurgeons use either frame-based ${ }^{13,19}$ or frameless ${ }^{1,2,15}$ stereotactic localization systems. Although frame-based stereotactic interventions are considered the gold standard for precise brain biopsies, they have limitations with regard to flexibility and patient comfort because of the need to attach a bulky head ring to the patient. ${ }^{8}$ In contrast, frameless imageguided systems, which use scalp fiducial markers, offer greater flexibility and patient comfort but provide less stability and accuracy during drilling and biopsy needle positioning. ${ }^{6}$

At some centers, safety and efficiency are further heightened by supplementing these localization systems with robotic probe holders, which decreases operator-introduced

SUBMITTED August 3, 2014. ACCEPTED December 8, 2014.

INCLUDE WHEN CITING Published online June 12, 2015; DOI: 10.3171/2014.12.JNS141781.

DISCLOSURE The authors report no conflict of interest concerning the materials or methods used in this study or the findings specified in this paper. The following grants funded this study: Dr. Gharabaghi was supported by the German Research Council (Grants DFG GH 94/2-1 and DFG EC 307) and the Federal Ministry for Education and Research (Grants BFNT 01GQ0761, BMBF 16SV3783, BMBF 03160064B, and BMBF V4UKF014). The Renaissance Surgical Guidance Robot for this study was provided by Mazor Robotics Ltd. (Caesarea, Israel).

* Drs. Grimm and Naros contributed equally to this work. 
variability. ${ }^{4}$ In recent years, robotic approaches have been developed to assist with brain surgeries. Although these technologies have added mechanization to the process, they still rely on either a stereotactic frame or frameless optical systems for intraoperative registration. The Programmable Universal Machine for Assembly (PUMA, Advanced Research \& Robotics) used a frame-based approach, and when it was introduced in 1985, it was the first reported robot for use in brain surgeries. ${ }^{10}$ Two years later, the Neuromate (Renishaw) was the first neurorobotic device to be approved by the FDA and the first to be commercially available. ${ }^{11}$ Minerva (University of Lausanne), introduced in 1991, relied on real-time image guidance and provided frameless stereotaxy. ${ }^{7}$ However, because it could perform only single-dimension incursions and the patient had to be inside a CT scanner, the project was discontinued in 1993. Several other robotic systems were designed and tested, and some were marketed (e.g., SurgiScope; Elekta). However, these surgical robots have not successfully overcome the inherent limitations of current localization systems, because they are still susceptible to human interference errors during either manual setting of the frame (in frame-based approaches) or intraoperative registration (in frameless approaches). Moreover, the current robotic devices either are not portable and/or suffer from poor ergonomics/ease of use. Renaissance (Mazor Robotics Ltd.) was recently introduced as a novel robotic guidance system that uses a small platform connected directly to the patient's skull. In the present study, we investigated the feasibility and safety of this portable headmounted robotic device for use in brain biopsies.

\section{Methods \\ Patients}

This study was performed in parallel at 2 different neurosurgical departments at university hospitals in Tuebingen and Mainz in Germany. Each patient who underwent a diagnostic brain biopsy at the respective departments of Eberhard Karls University or Johannes Gutenberg University with this FDA-approved and Conformité Européenne-marked device (Renaissance Surgical Guidance Robot, Mazor Robotics Ltd.) between May 2013 and January 2014 was included, and their charts were reviewed retrospectively in accordance with the Declaration of Helsinki and the guidelines of the local ethics committee. We included a consecutive series of patients scheduled for a stereotactic biopsy (i.e., no patients or tumor types were excluded). For infratentorial lesions, we perform open (e.g., microscopic) biopsies; therefore, patients with these lesions were not included.

\section{Surgical Planning}

Before surgery, the robot's planning software was used to create fused images from various imaging modalities (e.g., MRI, CT) to chart the intracranial path for the biopsy needle and avoid eloquent regions and vasculature. The software then suggested multiple geometrically suitable locations for positioning the robot's mounting platform on the patient's skull (Fig. 1). On the day of surgery, this mounting platform was fixated to the patient's head after administering local anesthesia to the respective skin area

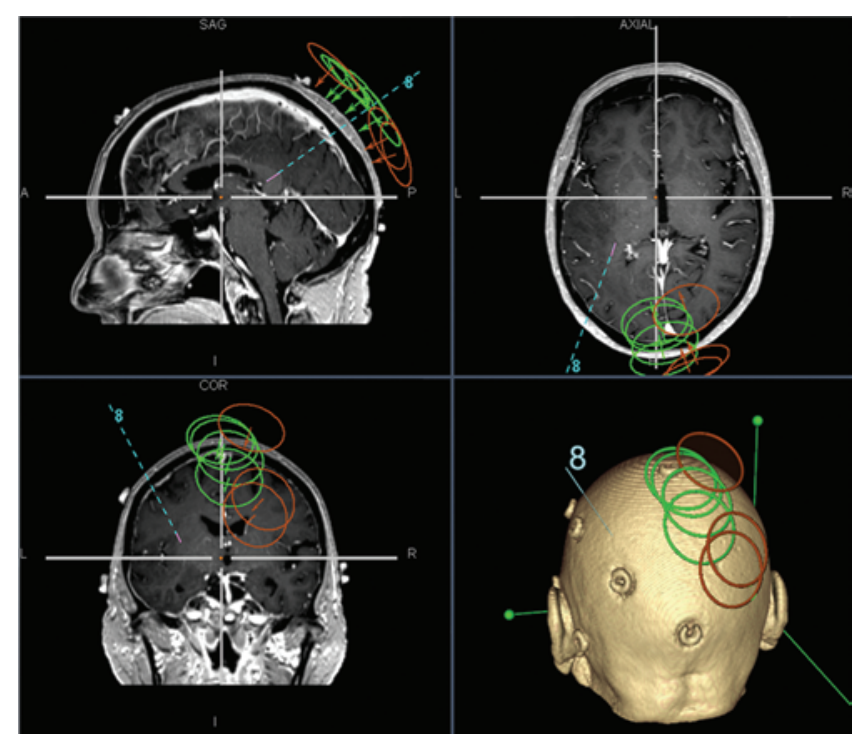

FIG. 1. Possible positions for the mounting platform and selected trajectory. Figure is available in color online only.

(Fig. 2). This procedure was performed at bedside with three $20.7-\mathrm{mm}$ screws, each penetrating $4 \mathrm{~mm}$ into the patient's skull. A 3D fiducial array was placed on top of this platform, and its final position relative to the patient's anatomy was determined by preoperative CT (1-mm-thick slices, multislice CT scanner [Siemens Medical GmbH]). The resulting CT image was fused with the planning images from other modalities, which usually included MR images (1-mm-thick slices, T1 3D dataset sequences [Siemens Medical $\mathrm{GmbH}]$ ), which created registration between the planned trajectories and the patient's anatomy with the physical platform temporarily attached to his or her head.

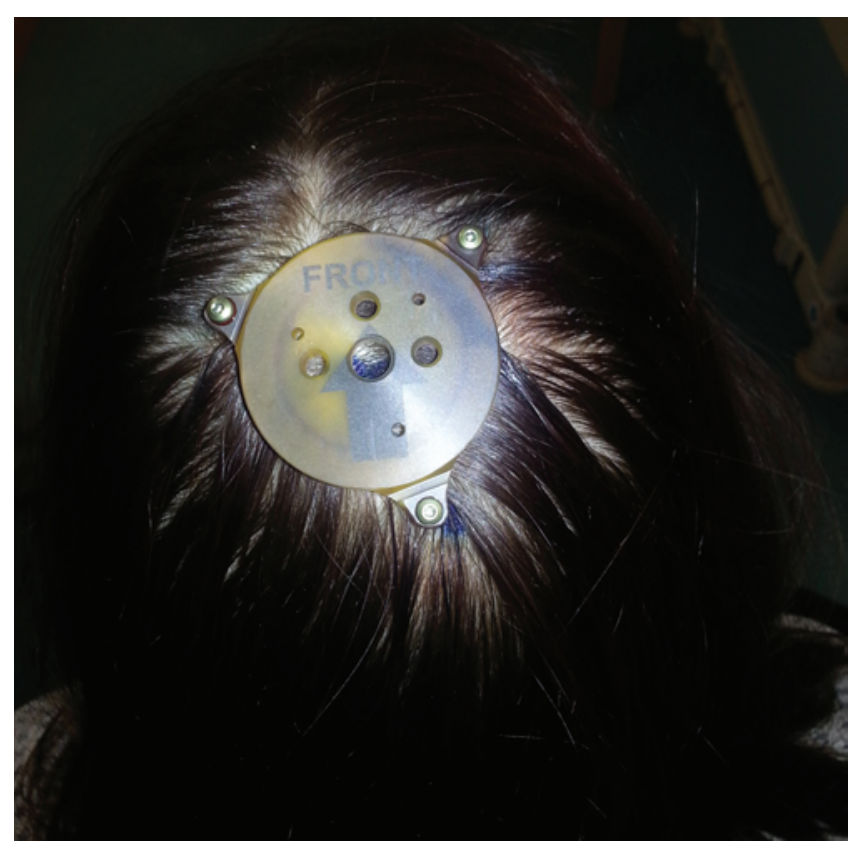

FIG. 2. Attachment of the mounting plate (performed after administering local anesthesia). Figure is available in color online only. 


\section{Set-Up}

The key component of the robotic guidance device is a cylindrical hexapod unit that measures $50 \times 90 \mathrm{~mm}$, weighs $400 \mathrm{~g}$, and has 6 degrees of freedom. This unit was attached to the skull-mounted reference platform (Fig. 3). The unit's working space is defined through a set of detachable arms with different ranges of reach. Based on coordinates predefined by the surgeon, the software automatically calculates optimal trajectories and aligns a guiding tube, through which the surgeon can insert tools such as the drill and biopsy needle.

\section{Procedure}

In each case, a standard frameless tracking system (VectorVision 3; Brainlab) was used in parallel with the robotic device to verify the trajectory. Every patient underwent surgery under general anesthesia. Before introducing the robotic device to the surgical field, a mechanical calibration process was performed. This calibration process included automatically sending the robot to 3 different set reference points on its workstation. The user then inserted a designated tool tip to the calibration arm to verify that the robot had reached the center of each point with $\leq 1.0$ $\mathrm{mm}$ accuracy. The system was cleared for intraoperative use only after reaching such accuracy. After patient positioning, the robotic device was placed in a sterile drape and attached to the head-mounted platform. Through a wired connection, the device was controlled by the mobile planning station. After the unit was attached to the skull, its predefined position and orientation were achieved in an

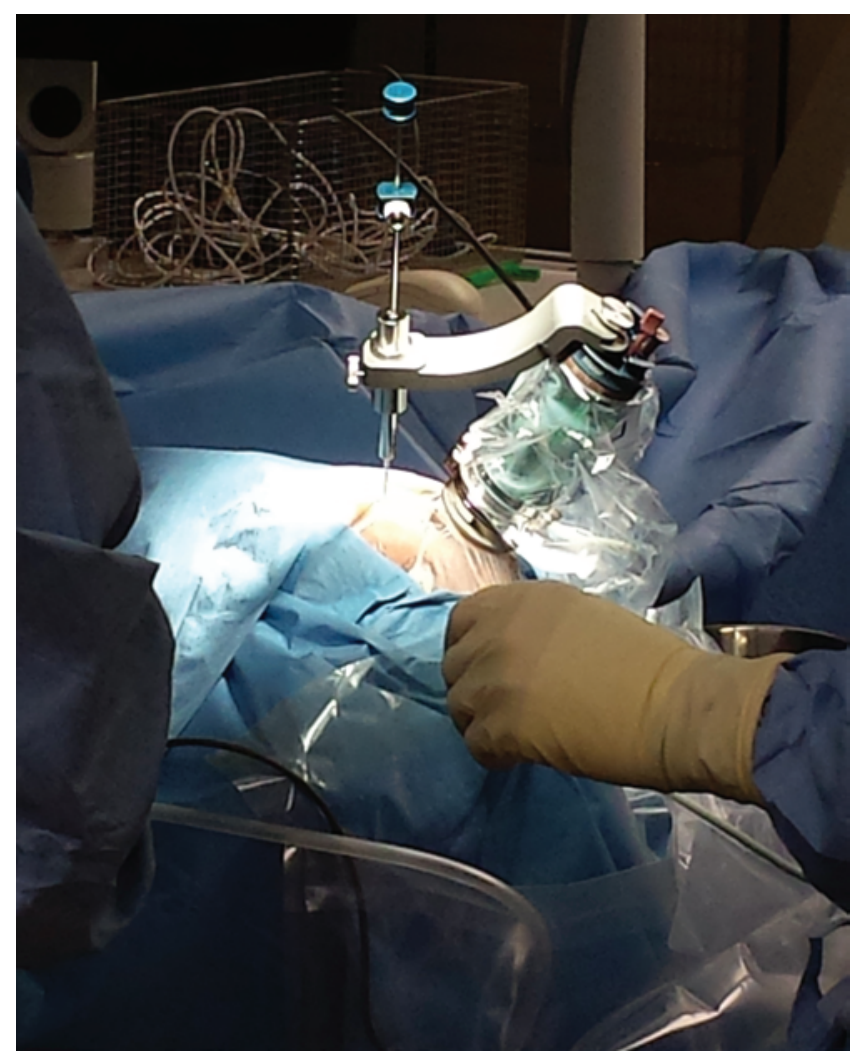

FIG. 3. Intraoperative set-up of the mounted hexapod unit. Figure is available in color online only. automated fashion. After this point, the robot functioned as a holder for standard instruments aligned with the chosen trajectory to facilitate needle biopsies. Because of the individual preferences of the surgeons, either a drill hole $(3 \mathrm{~mm})$ or a bur hole $(12 \mathrm{~mm})$ was created in 23 and 14 patients, respectively. Accordingly, dural incision and corticotomy were performed by either a stab or a microknife followed by monopolar or bipolar cauterization, respectively. The biopsies were performed with a negative-pressure technique in several quadrants at different depths. We did not observe back bleeding in any of the patients. The incision was closed according to individual preferences (e.g., with Gelfoam or fibrin glue) followed by bone cement whenever a bur hole was created. Postoperative CT scanning was performed for each patient within 24 hours after the operation to control for surgical complications.

\section{Results}

\section{Demographics and Lesion Characteristics}

Thirty-seven patients (mean age 57.4 years [range 1583 years]; 17 female) underwent a robotic biopsy. Lesions were located multifocally $(n=4)$, in the corpus callosum $(\mathrm{n}=7)$, in the basal ganglia $(\mathrm{n}=10)$, or in the central ( $\mathrm{n}$ $=4)$, frontal $(\mathrm{n}=2)$, parietal $(\mathrm{n}=4)$, temporal $(\mathrm{n}=4)$, or occipital $(n=2)$ region. The mean lesion diameter was $3.1 \mathrm{~cm}$ (range $0.7-7 \mathrm{~cm}$ ). Thirty-one of the lesions were contrast enhancing.

\section{Safety and Complications}

Postoperative CT images revealed an epidural hematoma in 1 patient that necessitated a craniotomy but resulted in no postoperative deficits. In this patient, a 3-mm drill hole had been created during the biopsy procedure followed by a stab incision of the dura. After this incident, most surgeons created a 12-mm bur hole in the following cases to achieve better visualization and allow for microknife incision of the dura. We observed no hemorrhages along the needle tracts, but minor hemorrhagic changes were seen at the biopsy site in 8 cases; none of them induced any space-occupying effect. One patient with a lesion in the frontal language area experienced transient postoperative deterioration of preexisting dysphasia. No persistent neurological deficits or infections were reported.

\section{Histology and Diagnostic Yield}

The histological diagnoses included 22 gliomas (16 glioblastomas, 5 anaplastic astrocytomas, and 1 low-grade glioma), 6 lymphomas, 1 bacterial abscess, and 5 inflammatory changes; in 3 cases, frozen-section histological analyses failed to reveal any disorder. For 1 cystic case, analysis of multiple samples failed to yield any diagnosis during the intraoperative neuropathological examination; this failure necessitated conversion to open biopsy during the surgery, which finally revealed a high-grade glioma.

\section{Feasibility and Handling}

The head-mounted platform was well tolerated by the patients and was positioned next to the incision site in all 
cases, thereby allowing flexible patient positioning during surgery. By attaching the robot directly to the headmounted platform, no additional manual registration of the tool was necessary. Moreover, the set-up was independent of maintaining a line of sight, which is usually necessary with frameless optical navigation systems. The robotic arm holder enabled stable drilling and needle positioning along the selected trajectories, even when they were very tangential to the surface of the skull (Fig. 4). The median time from skin incision to taking the first sample was 24 minutes (range 10-45 minutes); this time included the setup of the robotic system. For 2 patients with multifocal tumors, we preoperatively planned several trajectories for taking samples from different regions. These different trajectories were achieved easily by the robot without requiring manual calibrations, registration, or additional parameter settings by the surgeon (Fig. 5). In 5 other cases, the frame-based approach used at our institution would have failed to achieve the trajectory that was defined preoperatively on the working station because of the limited range of motion of the lateral arc angle $\left(65^{\circ}\right)$ of the CRW frame. In each of these cases the robot succeeded in achieving the very same trajectory (Fig. 6). In 2 cases, preoperative CT imaging had to be repeated (i.e., a deviation from the standard protocol was required); in 1 case the software did not recognize the registration platform in the first imaging attempt, and in the other case it was not possible to read the CT data into the system because of the specific Digital Imaging and Communications in Medicine (DICOM) settings of the scanner.

\section{Discussion}

To achieve maximal targeting precision, stereotactic surgery traditionally uses a rigid frame affixed to the patient's head during both imaging and surgical intervention. ${ }^{18}$ This technique prevents the need for intraoperative registration and thus increases overall accuracy. Conversely, these stereotactic frames necessitate manual parameter settings by the surgeon according to preoperative planning and thus can introduce human and calculation errors.12 Moreover, the frame is large and bulky, uncomfortable for

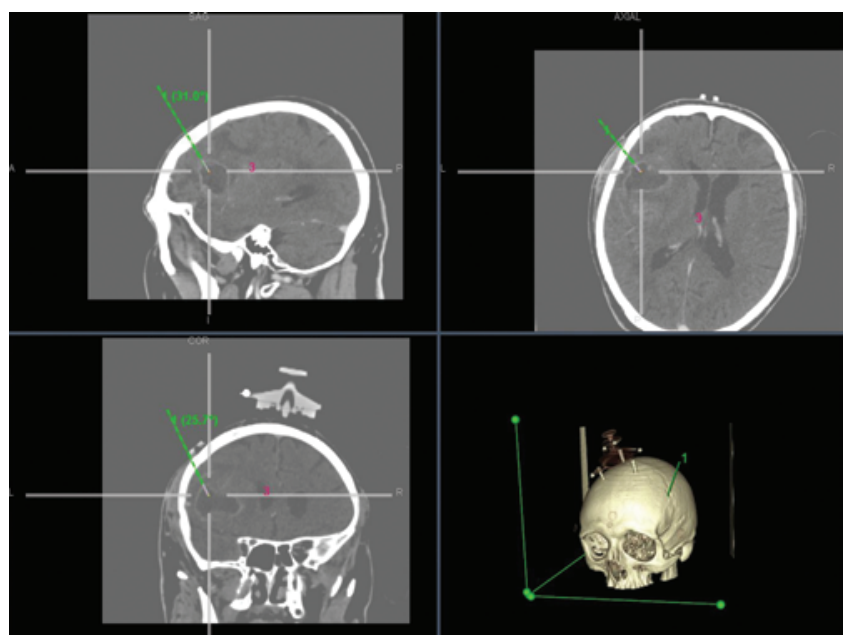

FIG. 4. Tangential trajectory planning for biopsy of a glioblastoma. Figure is available in color online only.

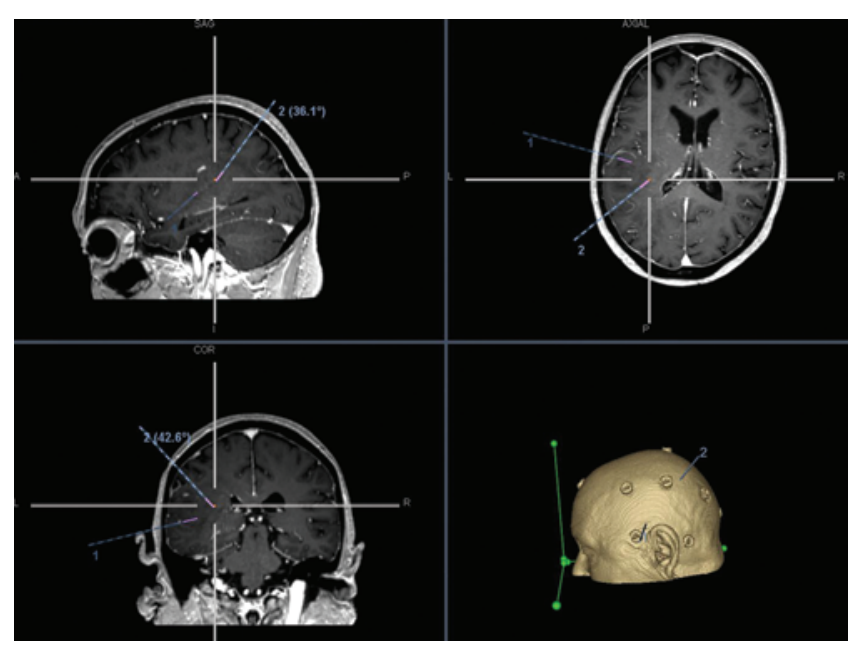

FIG. 5. Multiple predefined trajectories. Figure is available in color online only.

the patient, and cumbersome for the surgeon. With the introduction of frameless systems based on optical tracking technology, several limitations of frame-based systems were overcome, especially those regarding patient comfort and operative handling., ${ }^{3,15,17}$ Conversely, these frameless interventions depend on the surgeon's hand-eye coordination, thereby potentially introducing inaccuracies. ${ }^{5,16}$ Accordingly, although both frame-based and frameless approaches provide very high localization accuracy in the laboratory setting, their accuracy decreases significantly when applied in a clinical setting. ${ }^{12}$ Consequently, medical robots have been introduced to counter some of these limitations. ${ }^{14}$ While eliminating the error-susceptible step of manual setting, they provide accurate, predefined, and reprogrammable paths, hold instruments in place for repetitive tasks without tremor or fatigue, and perform any task with the same precision. ${ }^{4}$ Nonetheless, these systems still either use a stereotactic frame or apply frameless optical systems for intraoperative registration, thereby maintaining the inherent limitations of current localization systems (i.e., targeting tools are still operator dependent to a certain degree).

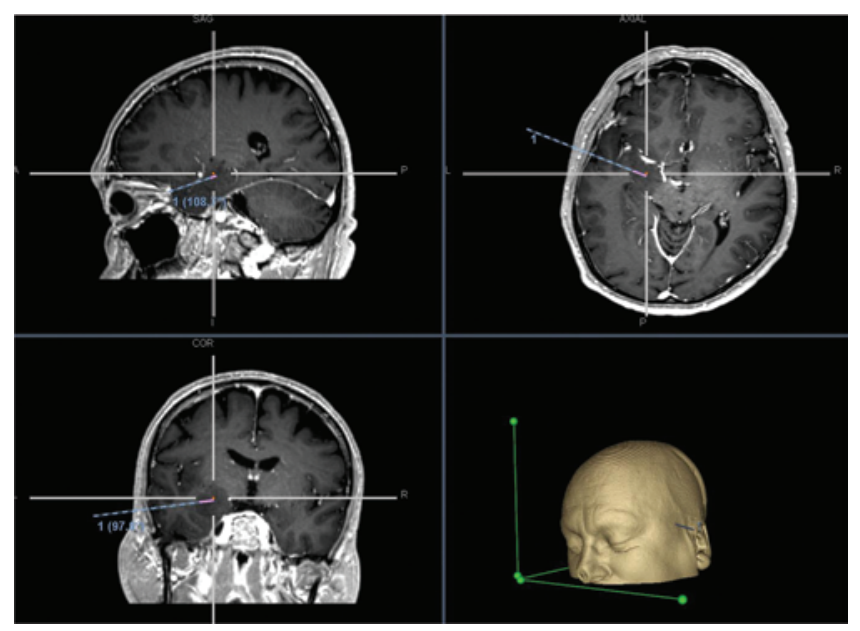

FIG. 6. Lateral trajectory for biopsy of a temporal low-grade glioma. Figure is available in color online only. 
To overcome some of these limitations, recent technological developments have implemented portable headmounted devices for trajectory-based procedures. Nexframe (Medtronic SNT) is a holding device that is attached to the patient's skull with a frame much smaller and lighter than classic frames. ${ }^{3}$ Although it can be adjusted manually during surgery, it provides no navigational guidance itself and necessitates guidance by an additional frameless tracking system such as the StealthStation (Medtronic SNT). Similarly, STarFix (FHC, Inc.) is a portable system that is also attached to the patient's skull, but in contrast to Nexframe, it uses its own navigational guidance. However, it provides limited flexibility and requires lengthy manufacturing time because it is custom made for each patient and each target. Very recently, a head-mounted robotic targeting device that is intraoperatively adjustable has been studied in head phantoms. ${ }^{9}$

To the best of our knowledge, this is the first clinical case series of patients undergoing trajectory-based intracranial interventions with the use of a portable headmounted robotic targeting device. This novel tool was easy to handle; that is, unlike in frame-based approaches, only 1 person was necessary to fix the device on the patient's skull. Moreover, there was no need to fix anything to the forehead of the patient, which is a feature of frame-based systems that is perceived by many patients as particularly uncomfortable. In contrast, the skull-mounted reference platform of the robot is rather flexible, in that it can be located next to the lesion. Each patient remained comfortably in bed during fixation of the reference platform, no hair shaving was necessary, and the application of local anesthesia was limited to one area; the whole presurgical part of the stereotactic intervention, which is often regarded as particularly stressful for patients, was well tolerated in each case. However, CT scans had to be acquired preoperatively because the 3D fiducial array, which is placed on top of the mounting platform for localization, was not MRI compatible.

The intraoperative procedure was safe because stable drilling and needle positioning could be performed without the need for constant verification on image guidance. Moreover, with this robotic approach, even very lateralbasal trajectories, which are not accessible with a standard stereotactic frame, could be achieved. However, the overall working volume and reachable distance of the robotic system was confined because only 1 reference platform could be mounted at a time and the length of the extension arms was limited, which necessitated aligned placement of the reference platform beforehand. At the same time, the proximity of the skull-mounted reference platform to the target region allowed for flexible patient positioning in the operating room independent of the working volume of ground- or ceiling-based robots. Moreover, based on the principle of shared control, flexible adjustments of predefined paths and selection of new trajectories were performed in an automated fashion during the procedure without needing device-related calibrations or adjustments. Thus, the surgeon was able to focus on the neurosurgical procedure and avoid manual frame setting and intraoperative registration.

This study is limited because it lacked an image-based measure of accuracy. As a result of patient preferences, no markers that could be detected on postoperative images were placed at the biopsy sites in these cases. Moreover, indirect signs such as the biopsy cavity or air trapping were too heterogeneous to serve as reliable markers for a precise comparison with the planned trajectory. Therefore, future studies will have to clarify the intraoperative accuracy of head-mounted robot-guided biopsies in comparison with that of standard frame-based and frameless procedures.

\section{Conclusions}

The presented head-mounted robot-guided approach combines the stability of a bone-mounted set-up with the flexibility of frameless systems. Moreover, flexible adjustments of predefined paths in an automated fashion during the procedure without the need for device-related adjustments or for constant verification on image guidance reduce the need for human interference via manual parameter settings by the surgeon (in frame-based stereotaxy), and dependence on a surgeon's hand-eye coordination (in frameless stereotaxy) can be avoided.

\section{References}

1. Air EL, Leach JL, Warnick RE, McPherson CM: Comparing the risks of frameless stereotactic biopsy in eloquent and noneloquent regions of the brain: a retrospective review of 284 cases. J Neurosurg 111:820-824, 2009

2. Amin DV, Lozanne K, Parry PV, Engh JA, Seelman K, Mintz A: Image-guided frameless stereotactic needle biopsy in awake patients without the use of rigid head fixation. $\mathbf{J}$ Neurosurg 114:1414-1420, 2011

3. Barbre CJ: Devices for targeting the needle. Neurosurg Clin N Am 20:187-191, 2009

4. Bekelis K, Radwan TA, Desai A, Roberts DW: Frameless robotically targeted stereotactic brain biopsy: feasibility, diagnostic yield, and safety. J Neurosurg 116:1002-1006, 2012

5. Dorward NL, Alberti O, Palmer JD, Kitchen ND, Thomas DG: Accuracy of true frameless stereotaxy: in vivo measurement and laboratory phantom studies. Technical note. J Neurosurg 90:160-168, 1999

6. Dorward NL, Paleologos TS, Alberti O, Thomas DG: The advantages of frameless stereotactic biopsy over frame-based biopsy. Br J Neurosurg 16:110-118, 2002

7. Glauser D, Fankhauser H, Epitaux M, Hefti JL, Jaccottet A: Neurosurgical robot Minerva: first results and current developments. J Image Guid Surg 1:266-272, 1995

8. Kitchen ND, Lemieux L, Thomas DG: Accuracy in framebased and frameless stereotaxy. Stereotact Funct Neurosurg 61:195-206, 1993

9. Kratchman LB, Fitzpatrick JM: Robotically-adjustable microstereotactic frames for image-guided neurosurgery. Proc SPIE 8671:86711U, 2013

10. Kwoh YS, Hou J, Jonckheere EA, Hayati S: A robot with improved absolute positioning accuracy for CT guided stereotactic brain surgery. IEEE Trans Biomed Eng 35:153-160, 1988

11. Li QH, Zamorano L, Pandya A, Perez R, Gong J, Diaz F: The application accuracy of the NeuroMate robot-a quantitative comparison with frameless and frame-based surgical localization systems. Comput Aided Surg 7:90-98, 2002

12. Maciunas RJ, Galloway RL Jr, Latimer JW: The application accuracy of stereotactic frames. Neurosurgery 35:682-695, 1994 
13. Owen CM, Linskey ME: Frame-based stereotaxy in a frameless era: current capabilities, relative role, and the positiveand negative predictive values of blood through the needle. $\mathbf{J}$ Neurooncol 93:139-149, 2009

14. Shoham M, Burman M, Zehavi E, Joskowicz L, Batkilin E, Kunicher Y: Bone-mounted miniature robot for surgical procedures: concept and clinical applications. IEEE Trans Rob Autom 19:893-901, 2003

15. Smith JS, Quiñones-Hinojosa A, Barbaro NM, McDermott MW: Frame-based stereotactic biopsy remains an important diagnostic tool with distinct advantages over frameless stereotactic biopsy. J Neurooncol 73:173-179, 2005

16. Villalobos H, Germano IM: Clinical evaluation of multimodality registration in frameless stereotaxy. Comput Aided Surg 4:45-49, 1999

17. Widmann G, Schullian P, Ortler M, Bale R: Frameless stereotactic targeting devices: technical features, targeting errors and clinical results. Int J Med Robot 8:1-16, 2012

18. Willems PW, van der Sprenkel JW, Tulleken CA, Viergever MA, Taphoorn MJ: Neuronavigation and surgery of intracerebral tumours. J Neurol 253:1123-1136, 2006

19. Woodworth G, McGirt MJ, Samdani A, Garonzik I, Olivi A,
Weingart JD: Accuracy of frameless and frame-based imageguided stereotactic brain biopsy in the diagnosis of glioma: comparison of biopsy and open resection specimen. Neurol Res 27:358-362, 2005

\section{Author Contributions}

Conception and design: Grimm, Naros, Gharabaghi. Acquisition of data: all authors. Analysis and interpretation of data: Grimm, Naros, Gharabaghi. Drafting the article: Gharabaghi. Critically revising the article: all authors. Reviewed submitted version of manuscript: all authors. Approved the final version of the manuscript on behalf of all authors: Gharabaghi. Statistical analysis: Grimm, Naros. Administrative/technical/material support: all authors. Study supervision: Gharabaghi.

\section{Correspondence}

Alireza Gharabaghi, Division of Functional and Restorative Neurosurgery, Department of Neurosurgery, Eberhard Karls University, Otfried-Mueller-Strasse 45, 72076 Tuebingen, Germany. email: alireza.gharabaghi@uni-tuebingen.de. 